\title{
Persona2vec: A flexible multi-role representations learning framework for graphs
}

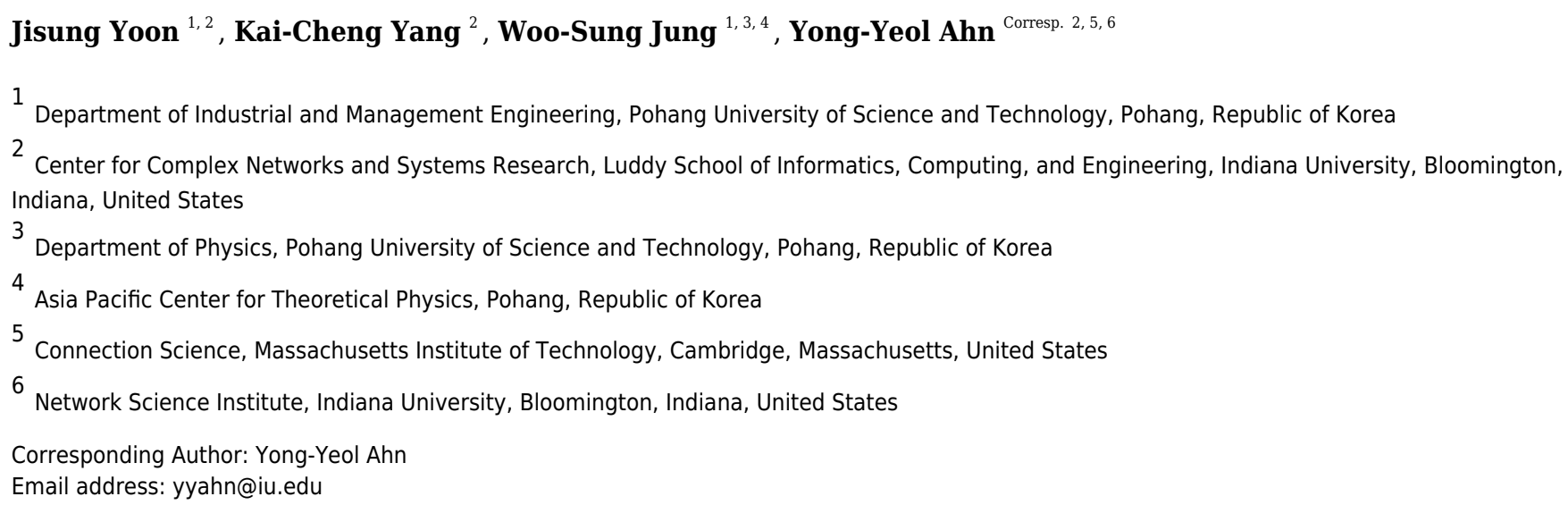

Graph embedding techniques, which learn low-dimensional representations of a graph, are achieving state-of-the-art performance in many graph mining tasks. Most existing embedding algorithms assign a single vector to each node, implicitly assuming that a single representation is enough to capture all characteristics of the node. However, across many domains, it is common to observe pervasively overlapping community structure, where most nodes belong to multiple communities, playing different roles depending on the contexts. Here, we proposepersona2vec, a graph embedding framework that efficiently learns multiple representations of nodes based on their structural contexts. Using link prediction-based evaluation, we show that our framework is significantly faster than the existing state-of-the-art model while achieving better performance. 


\title{
Persona2vec: A flexible multi-role
} - representations learning framework for - graphs

\author{
Jisung Yoon ${ }^{1,2}$, Kai-Cheng Yang ${ }^{2}$, Woo-Sung Jung ${ }^{1,3,4}$, and Yong-Yeol \\ $\mathrm{Ahn}^{2,5,6}$ \\ ${ }^{1}$ Department of Industrial and Management Engineering, Pohang University of Science \\ and Technology, Pohang 37673, Republic of Korea. \\ ${ }^{2}$ Center for Complex Networks and Systems Research, Luddy School of Informatics, \\ Computing, and Engineering, Indiana University, Bloomington, IN 47408, USA \\ ${ }^{3}$ Department of Physics, Pohang University of Science and Technology, Pohang 37673, \\ Republic of Korea. \\ ${ }^{4}$ Asia Pacific Center for Theoretical Physics, Pohang 37673, Republic of Korea. \\ ${ }^{5}$ Network Science Institute, Indiana University, Bloomington (IUNI), IN 47408, USA \\ ${ }^{6}$ Connection Science, Massachusetts Institute of Technology, Cambridge, MA 02139, \\ USA
}

Corresponding author:

Yong-Yeol Ahn ${ }^{1}$

Email address: yyahn@iu.edu

\section{ABSTRACT}

Graph embedding techniques, which learn low-dimensional representations of a graph, are achieving state-of-the-art performance in many graph mining tasks. Most existing embedding algorithms assign a single vector to each node, implicitly assuming that a single representation is enough to capture all characteristics of the node. However, across many domains, it is common to observe pervasively overlapping community structure, where most nodes belong to multiple communities, playing different roles depending on the contexts. Here, we propose persona2vec, a graph embedding framework that efficiently learns multiple representations of nodes based on their structural contexts. Using link predictionbased evaluation, we show that our framework is significantly faster than the existing state-of-the-art models while achieving better performance.

\section{INTRODUCTION}

Graph embedding maps the nodes in a graph to continuous and dense vectors that capture relations among the nodes (Perozzi et al., 2014; Grover and Leskovec, 2016; Tang et al., 2015). Resulting node representations allow direct applications of algebraic operations and common algorithms, facilitating graph mining tasks such as node classification (Sen et al., 2008; Perozzi et al., 2014), community detection (Fortunato, 2010; Yang et al., 2016), link prediction (Grover and Leskovec, 2016), visualization (Tang et al., 2015), and computer vision (Xie et al., 2020). Most methods map each node to a single vector, implicitly assuming that a single representation is sufficient to capture the full characteristics of a node.

However, nodes often play multiple roles. For instance, people have multiple roles, or "personas", across contexts (e.g. professor, employee, and so on) (Ahn et al., 2010; Coscia et al., 2014; Leskovec et al., 2009, 2010). Similarly, proteins and other biological elements play multiple functionalities (Palla et al., 2005; Gavin et al., 2006; Ahn et al., 2010). Another example is the polysemy of words when their relations are modeled with graphs; many words possess multiple meanings differentiated by the contexts (Chen et al., 2014; Li and Jurafsky, 2015; Iacobacci et al., 2015). Explicit modeling of such multiplicity and overlapping clusters has been fruitful not only for community detection (Rosvall et al., 2014; Coscia et al., 2014; Epasto et al., 2017), but also for improving the quality of embedding (Li and Jurafsky, 2015; 
Epasto and Perozzi, 2019; Liu et al., 2019). Yet, with the scarcity of embedding methods embracing this idea, the full potential of this approach has not been properly explored.

In this paper, we propose persona $2 \mathrm{vec}$, a scalable framework that builds on the idea of ego-splitting (Epasto et al., 2017), the process of identifying local structural contexts of a node via performing local community detection on the node's ego-network. For each detected local community (role), we transform each node into multiple personas if there are multiple local communities to which the node belongs. After the split, the original node is replaced by the new persona nodes that inherit the connection from each local community, producing a new persona graph. Instead of separating a node's persona nodes from each other completely (Epasto and Perozzi, 2019), we add directed, weighted edges between personas to capture their origin. In doing so, we allow the direct application of the existing graph embedding methods. In addition, we take an approach of considering persona-based learning as fine-tuning of the base graph embedding, achieving both efficiency and balance between information from the original graph and the persona graph. Compared with the previous approach (Epasto and Perozzi, 2019), our framework is conceptually simpler to understand and practically easier to implement. Furthermore, it achieves better performance in the link prediction tasks while being much faster. We also would like to clarify that the primary purpose of persona splitting is not about obtaining multiple representations, each of which may be suited for a specific task; it is about teasing out multiple contexts that a single node may possess. In other words, even with a single task, we argue that learning multiple representations for some nodes are highly beneficial.

In sum, we would like to highlight that our approach (1) drastically lowers the barrier for combining existing algorithms with persona splitting, (2) significantly improves the efficiency of the ego-splitting approach, while (3) consistently excelling the previous state-of-the-art model in the link prediction task. Our implementation of persona2vec is publicly available at https://github.com/jisungyoon/ persona2vec.

\section{RELATED WORK}

In addition to graph embedding, our work is closely related to the research of identifying overlapping communities in graphs. Various non-embedding methods such as link clustering (Ahn et al., 2010; Evans and Lambiotte, 2009), clique percolation (Palla et al., 2005), and mixed membership stochastic blockmodel (Airoldi et al., 2008) have been proposed. Another thread of works focuses on using local graph structure to extract community information (Coscia et al., 2014; Epasto et al., 2015, 2017). Specifically, Epasto et al. introduce the persona graph method for detecting overlapping communities in graphs (Epasto et al., 2017), leveraging ego-network partition. The combination of ego-network analysis and graph embedding methods is still rare. An example is SPLITTER (Epasto and Perozzi, 2019), which we use as the baseline in this paper. Instead of constraining the relations between personas with a regularization term, we propose a simpler and more efficient way of adding persona edges to the graph.

Our work is also related to the word disambiguation problem in a word embedding. Recently, word embedding techniques (Mikolov et al., 2013a,b; Pennington et al., 2014) have been extensively applied to various NLP tasks as the vectorized word representations can effectively capture syntactic and semantic information. Although some words have multiple senses depending on the context, the original word embedding methods only assign one vector to each word. Li et al. shows that embedding that is aware of multiple word senses and provides vectors for each specific sense does improve the performance for some NLP tasks (Li and Jurafsky, 2015). For this issue, some utilize the local context information and clustering for identifying word sense (Reisinger and Mooney, 2010; Wu and Giles, 2015; Neelakantan et al., 2015), some resort to external lexical database for disambiguation (Rothe and Schütze, 2015; Iacobacci et al., 2015; Camacho-Collados et al., 2016; Chen et al., 2014; Jauhar et al., 2015; Pelevina et al., 2017), while some combine topic modeling methods with embedding (Liu et al., 2015b,a; Cheng et al., 2015; Zhang and Zhong, 2016). We adopt the idea of assigning multiple vectors to each node in the graph to represent different roles as well as exploiting local graph structure for the purpose.

\section{PROPOSED METHOD: PERSONA2VEC}

persona2vec creates a persona graph, where some nodes are split into multiple personas. We then apply a graph embedding algorithm to the persona graph to learn the embeddings of the personas (see Fig. 1). Let us explain the method formally. Let $G=(V, E)$ be a graph with a set of nodes $V$ and a 
(a) Original graph

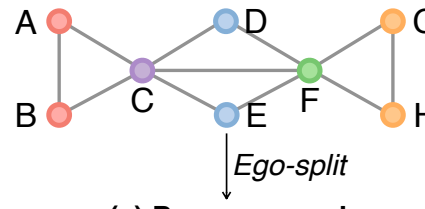

(c) Persona graph

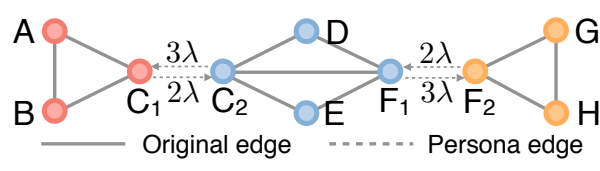

(b) Base graph embedding

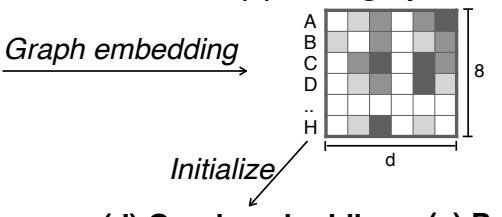

(d) Graph embedding

(e) Persona embedding
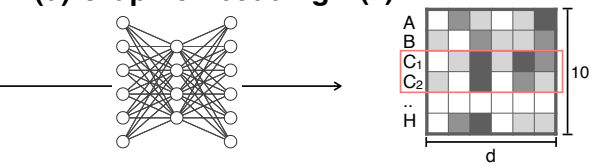

Figure 1. Illustration of persona2vec framework. (a) A graph with an overlapping community structure. (b) Graph embedding of the original graph is obtained first to initialize the persona embeddings. (c) Transform the original graph into a persona graph. Every edge in the original graph is preserved in the persona graph, while new directed persona edges with weight $\lambda k_{i}^{0}$ are added between the persona nodes. (d) Graph embedding is applied to the persona graph. (e) The final persona embedding where each persona node has its own vector representation.

set of edges $E .|V|$ and $|E|$ denote the number of nodes and edges respectively. Let $f: v \rightarrow \mathbb{R}^{d}$ be the embedding function that maps a node $v$ to a $d$-dimensional vector space $(d \ll|V|)$.

\section{Refined Ego-splitting}

We adopt and refine the ego-splitting method (Epasto et al., 2017; Epasto and Perozzi, 2019). For each node in the original graph, we first extract its ego-graph, remove the ego, and identify the local clusters. Every cluster in the ego-graph leads to a new persona node in the persona graph (see Fig. 1a, c). For example, if we consider each connected component as a local community with a connected component algorithm, node $C$ in the original graph belongs to two non-overlapping clusters $\{A, B\}$ and $\{D, E, F\}$ in its ego-graph. Given these two clusters, in the persona graph, $C$ is split into $C_{1}$ and $C_{2}$ to represent the two roles in respective clusters. $C_{1}$ and $C_{2}$ inherit the connections of $C$ from both clusters separately (see Fig. 1c). On the other hand, node $A$ only belongs to one ego cluster $\{B, C\}$, so it does not split into multiple personas.

Any graph clustering algorithm can be employed for splitting a node into personas. The simplest algorithm is considering each connected component in the ego-network (sans the ego) as a cluster. This approach is fast and works well on sparse graphs. However, in dense graphs, ego-networks are more likely to form fewer connected component, thus other algorithms such as the Louvain method (Blondel et al., 2008), Infomap (Rosvall and Bergstrom, 2008), and label propagation (Raghavan et al., 2007) would be more appropriate.

In previous studies, the personas get disconnected without retaining the information about their origin, creating isolated components in the splitting process (Epasto et al., 2017; Epasto and Perozzi, 2019). Because of this disconnectedness, common embedding methods could not be directly applied to the splitted graph. A previous study attempted to address this issue by imposing a regularization term in the cost function to penalize separation of persona nodes originating from the same node (Epasto and Perozzi, 2019).

Here, instead of adopting the regularization strategy, we add weighted persona edges between the personas, maintaining the connectedness between them after the splitting (see Fig. 1c). Because the persona graph stays connected, classical graph algorithms and graph embedding methods can now be readily applied without any modification. As we will show later, our strategy achieves both better scalability and better performance.

In the persona graph, we set the weights of the unweighted original edges as 1 and tune the strength of the connections among personas with $\lambda$. Persona edges are directed and weighted, with weight $\lambda k_{i}^{0}$, where $k_{i}^{0}$ is the out-degree of the persona node after splitting (see Fig. 1c). Assigning weight proportional to $k_{i}^{\mathrm{o}}$ helps the random walker exploring both the local neighbors and other parts of the graph connected to the other personas regardless of its out-degree $k_{i}^{\text {o }}$.

Imagine node $u$, which is split into $n_{p}$ personas. Consider one of the personas $i$ with out-degree $k_{i}^{\mathrm{o}}$ 


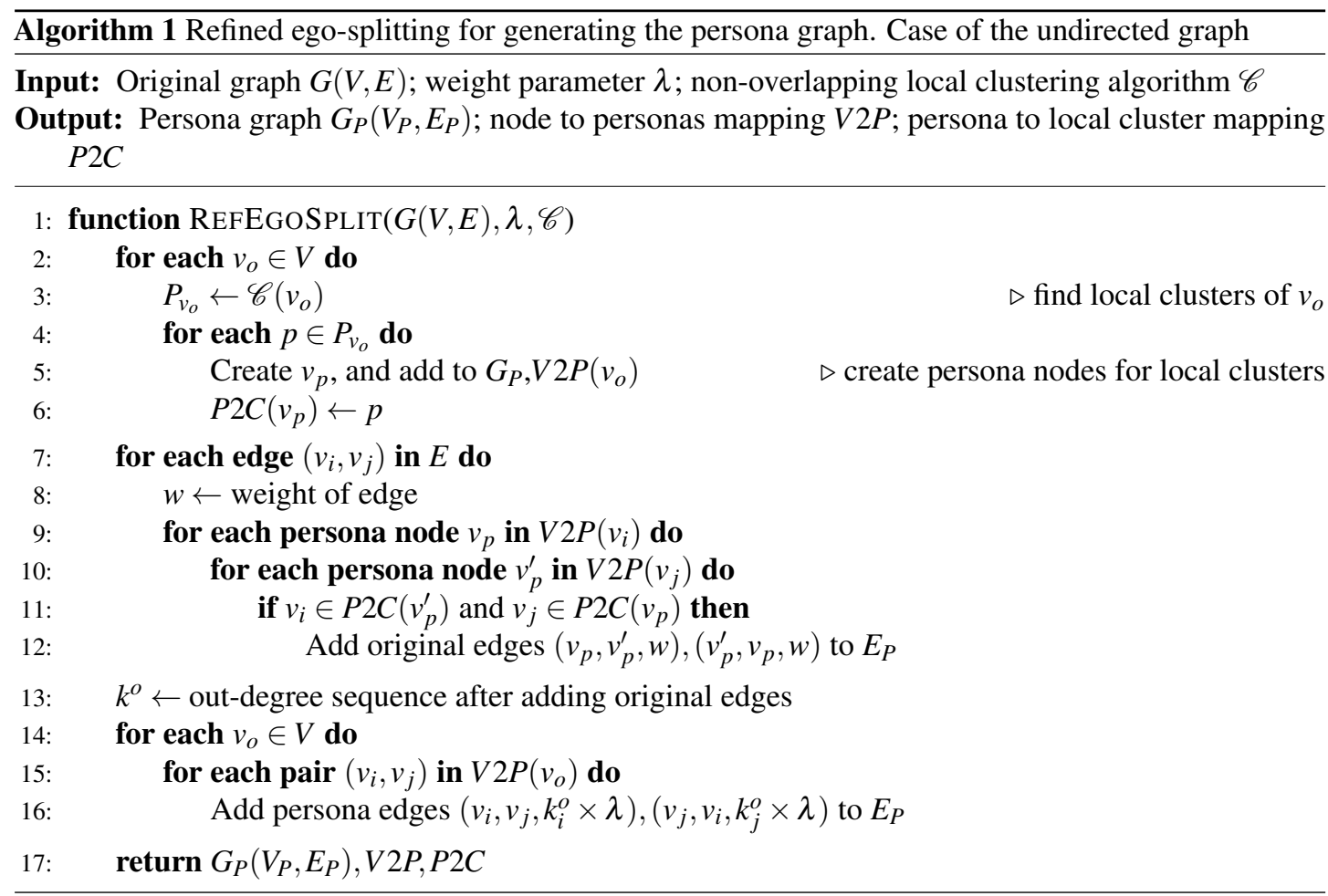

and persona edges with weight $w_{i}$. Then the probability $p_{i}$ that an unbiased random walker at $i$ visits neighbors connected with the original edge at the next step is

$$
\frac{k_{i}^{\mathrm{o}}}{k_{i}^{\mathrm{o}}+n_{p} w_{i}} .
$$

If we set constant weight $w_{i}=\lambda$, then

$$
p_{i}=\frac{k_{i}^{\mathrm{O}}}{k_{i}^{\mathrm{O}}+n_{p} \lambda}=\frac{1}{1+\frac{n_{p}}{k_{i}^{\mathrm{o}}} \lambda},
$$

which depends on $k_{i}^{\mathrm{o}}$. A random-walker would not explore its local neighborhood if $n_{p} \gg k_{i}^{\mathrm{o}}$, while the opposite happens when $n_{p} \ll k_{i}^{\mathrm{o}}$. Instead, assigning the weight proportional to $k_{i}^{\mathrm{o}}$, namely $w_{i}=\lambda k_{i}^{\mathrm{o}}$, removes such bias because

$$
p_{i}=\frac{k_{i}^{\mathrm{o}}}{k_{i}^{\mathrm{o}}+n_{p} \lambda k_{i}^{\mathrm{o}}}=\frac{1}{1+n_{p} \lambda},
$$

which is independent of $k_{i}^{\mathrm{o}}$. Our experiments also show that using the out-degree yields better performance than assigning the identical weight to each persona edge. Our algorithm for refined ego-splitting is described in Algorithm 1. Note that it can be generalized to the directed graphs.

\section{Persona graph embedding}

As explained above, any graph embedding algorithm that recognizes edge direction and weight can be readily applied to the persona graph. Although we use node $2 \mathrm{vec}$ as the embedding method here, other embedding methods can also be employed. We initialize the persona vectors with the vectors from the original graph before ego-splitting (see Fig. 1b) to leverage the information from the original graph structure. Persona nodes that belong to the same node in the original graph are thus initialized with the same vector. We then execute the embedding algorithm for a small number of epochs to fine-tune the embedding vectors with the information from the persona graph (see Fig. 1). Experiments show that usually only one epoch of training is enough. 


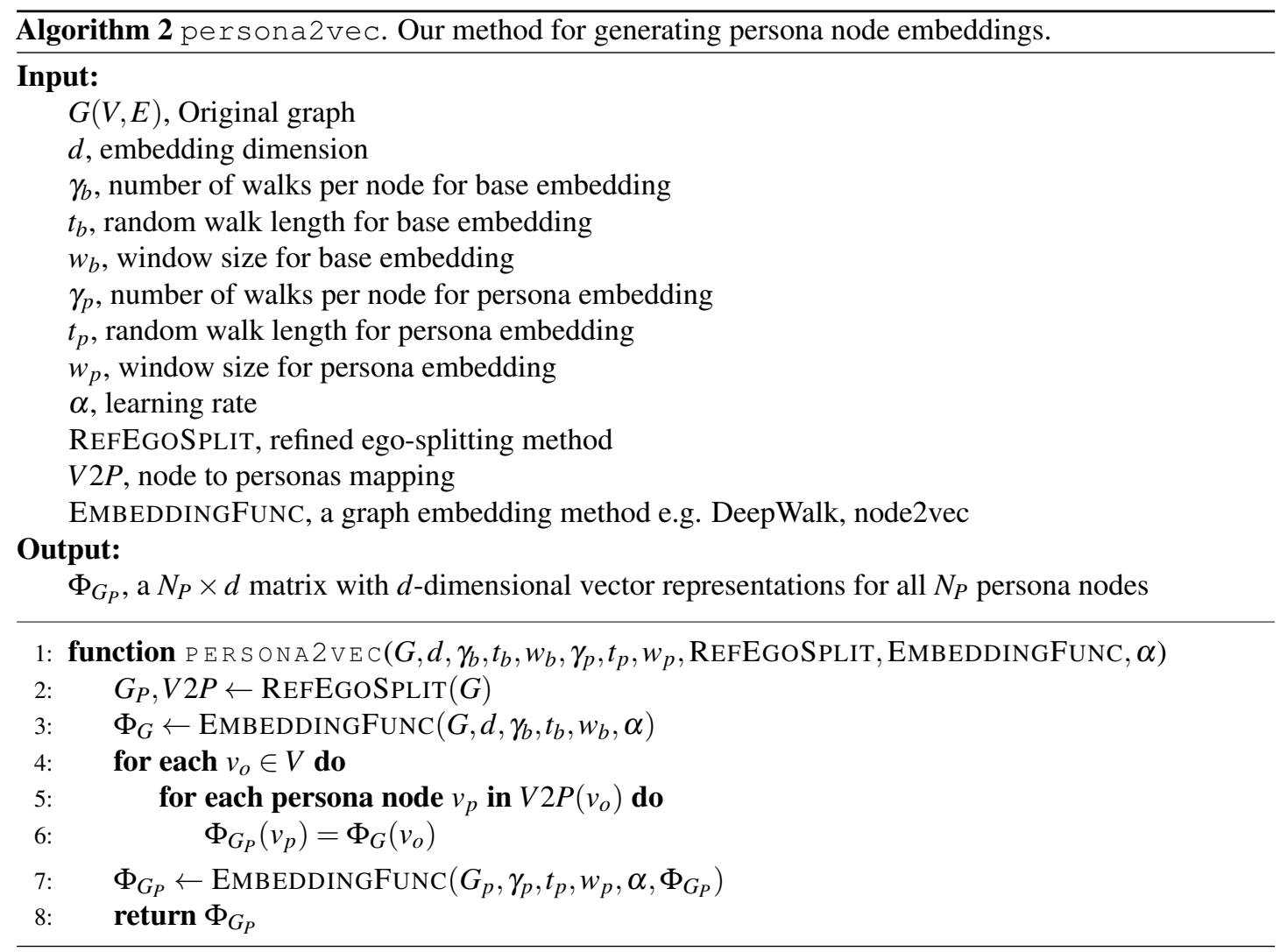

Also, training the embedding on the persona graphs from scratch fails to yield comparable results. We find that initializing the embedding with the original graphs, i.e., our present method, consistently improves the performance, suggesting that mixing the structural information from both the original graph and the persona graph is crucial. Our full algorithm is described in Algorithm 2.

\section{Complexity}

Space complexity The persona graph is usually larger than the original graph, but not too large. Node $u$ with degree $k_{u}$ may be split into at most $k_{u}$ personas. In the worst case, the number of nodes in the persona graph can reach $O(|E|)$. But, in practice, only a subset of nodes split into personas, and the number of personas rarely reaches the upper bound. If we look at the persona edges, for a node $u$ with degree $k_{u}$, at most $O\left(k_{u}^{2}\right)$ new persona edges may be added. Thus, the whole persona graph has at most $O\left(|V| \times k_{\max }^{2}\right)$ or $O\left(|V|^{3}\right)\left(\because k_{\max } \leq|V|\right)$ extra persona edges. If graph's degree distribution follows a power-law distribution $P(k) \sim k^{-\gamma}$, then $k_{\max } \sim|V|^{1 / \gamma-1}$. Hence, it could be $O\left(|V|^{\gamma+1 / \gamma-1}\right)$ and it is between $O\left(|V|^{2}\right)$ and $O\left(|V|^{3}\right)(\because 2 \leq \gamma \leq 3$ in general). However, real graph tends to be sparse and $k_{i} \ll|V|$. If we further assume $k_{i}<\sqrt{|E|}$ holds for every node, then $\sum_{n=1}^{|V|} k_{n}^{2} \leq \sum_{n=1}^{|V|} k_{n} \sqrt{|E|}=2|E| \sqrt{|E|}$. Under this assumption, the upper bound becomes $O\left(|E|^{3 / 2}\right)$. Similarly, with the scale-free condition, the upper bound could be $O\left(|E||V|^{1 / \gamma-1}\right)$, which is between $O\left(|E||V|^{1 / 2}\right)$ and $O(|E||V|)$. Again, in practice, the number of persona edges is much smaller than this upper bound. To illustrate, we list the number of nodes and persona edges in the persona graph for the graphs we use in this paper in Table 1. All considered, the extra nodes and edges do not bring too much space complexity burden in practice.

Time complexity Assessing the time complexity requires consideration of the two steps: ego-splitting and embedding. The ego-splitting algorithm has complexity of $O\left(|E|^{3 / 2}+\sqrt{|E|} T(|E|)\right)$ in the worst case, where $|E|$ is the number of edges in the original graph and $T(|E|)$ is the complexity of detecting the ego clusters in the graph with $|E|$ edges (Epasto et al., 2017). The embedding on the persona graph, which dominates the whole embedding procedure, has complexity $O\left(\left|V_{p}\right| \gamma t w d\left(1+\log \left(\left|V_{p}\right|\right)\right)\right)$ which is time complexity of Node2vec, where $\left|V_{p}\right|$ is the number of nodes, $\gamma$ is the number of random walkers, $d$ is the embedding dimension, and $w$ is the window size (Chen et al., 2018). 


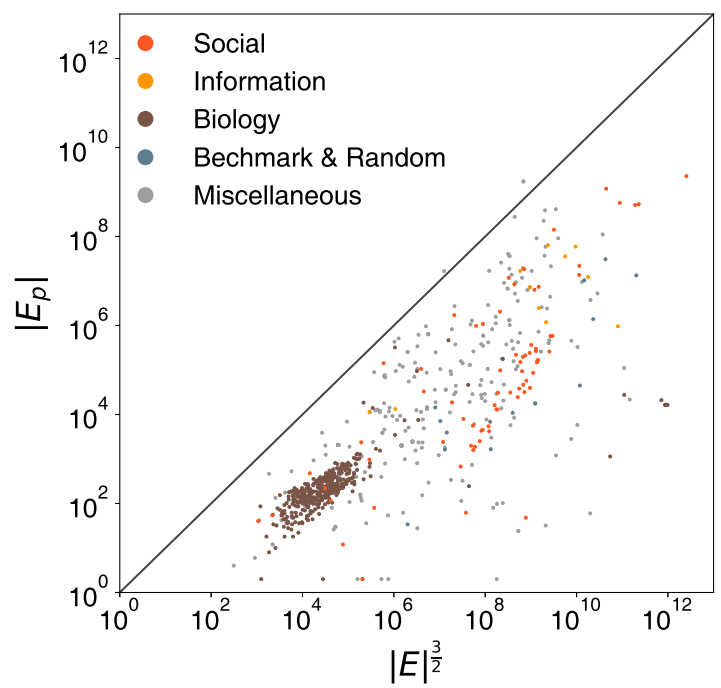

Figure 2. Comparison of the the number of persona edges $\left|E_{p}\right|$ to the practical upper bound $|E|^{3 / 2}$.

Table 1. Descriptive statistics in the graphs used in the evaluation. We report the number of nodes $|V|$, number of edges $|E|$, number of nodes in the persona graph $\left|V_{p}\right|$, the ratio of $\left|V_{p}\right|$ over $|V|$, number of persona edges $\left|E_{p}\right|$ added in ego-splitting, and the ratio of $\left|E_{p}\right|$ over $\left|E^{3 / 2}\right|$ which is the upper bound of space complexity.

\begin{tabular}{llrrrrrr}
\hline Dataset & Type & $|V|$ & $|E|$ & $\left|V_{p}\right|$ & $\left|V_{p}\right| /|V|$ & $\left|E_{p}\right|$ & $\left|E_{p}\right| /\left|E^{3 / 2}\right|$ \\
\hline PPI & Undirected & 3,863 & 38,705 & 16,734 & 4.34 & 132,932 & 0.0175 \\
ca-HepTh & Undirected & 9,877 & 25,998 & 16,071 & 1.86 & 33,524 & 0.0800 \\
ca-AstroPh & Undirected & 17,903 & 197,301 & 25,706 & 1.44 & 29,012 & 0.0003 \\
wiki-vote & Directed & 7,066 & 103,633 & 21,476 & 3.04 & 118,020 & 0.0035 \\
soc-epinions & Directed & 75,877 & 508,836 & 220,332 & 2.90 & $3,550,594$ & 0.0098 \\
\hline
\end{tabular}

The final complexity is $O\left(|E|^{3 / 2}+\sqrt{|E|} T(|E|)\right)+O(|V| \gamma t w d(1+\log (|V|)))$. Removing the constant factors and assuming close-to-linear local community detection algorithm, the whole process has time complexity of $O\left(|E|^{3 / 2}\right)$ with space complexity of $O\left(|E|^{3 / 2}\right)$ if $k_{i}<\sqrt{|E|}$ holds. Complexity can be increased depending on the clustering algorithms on the ego-network.

To test the validity of our assumptions, we sample 1,000 graphs from a public network repository (Rossi and Ahmed, 2015). We apply the refined ego-splitting with connected component algorithms on these samples and report the actual number of persona edges $\left|E_{p}\right|$ with respect to the practical upper bound $|E|^{3 / 2}$ in Fig. 2, which shows that the actual number of persona edges $\left|E_{p}\right|$ rarely exceeds the tighter upper bound that we proposed and is usually orders of the magnitude smaller.

\section{Optimization}

Any kind of graph embedding method can be considered, for simplicity, we choose the classical randomwalker based embedding method (e.g. Node2Vec, DeepWalk). In the model (Perozzi et al., 2014), the probability of a node $v_{i}$ co-occurring with a node $v_{j}$ is estimated by

$$
p\left(v_{i} \mid v_{j}\right)=\frac{\exp \left(\boldsymbol{\Phi}_{v_{i}}^{\prime} \cdot \boldsymbol{\Phi}_{v_{j}}\right)}{\sum_{k=1}^{V} \exp \left(\boldsymbol{\Phi}_{v_{k}}^{\prime} \cdot \boldsymbol{\Phi}_{v_{j}}\right)},
$$

where $\boldsymbol{\Phi}_{v_{i}}$ and $\boldsymbol{\Phi}_{v_{i}}^{\prime}$ are the "input" and "output" embedding of node $i$. We use input embedding $\boldsymbol{\Phi}$ which is known to be more useful and more widely used. Denominator of eq.4 is computationally expensive (Yang et al., 2016; Cao et al., 2016) and there are two common approximations: hierarchical softmax 
a

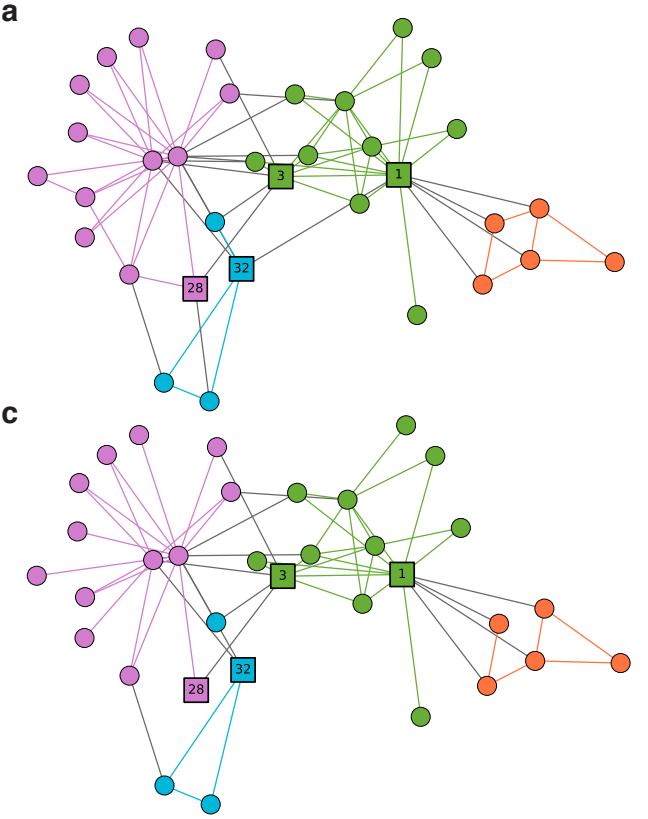

b

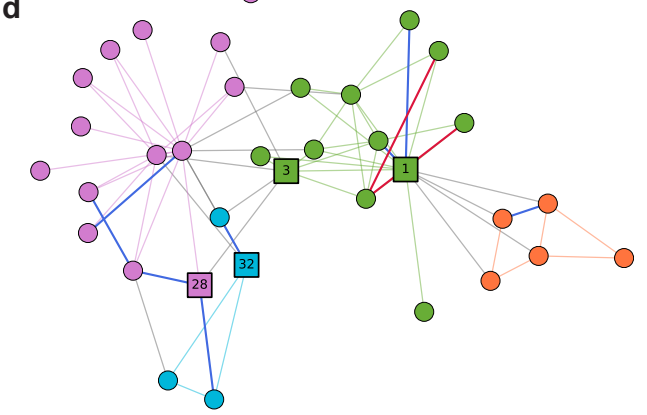

Figure 3. Case Study: Zachary's Karate club network (a) The Zachary's Karate club network with the force-atlas layout (Zachary, 1977). Nodes are colored by communities detected by the Louvain modularity method (Blondel et al., 2008). (b) The persona graph. Nodes are colored by k-means clusters (MacQueen et al., 1967) from the embedding vectors. Coordinates of the persona nodes come from the 2-D projection of the embedding with t-SNE (Maaten and Hinton, 2008). Light grey lines represent the persona edges. (c) The network with $20 \%$ of edges (16 edges) removed for the link prediction experiment. (d) The network with ten predictions with the highest score from the link prediction experiment. Blue links represent correctly predicted edges and red edges indicate incorrectly predicted ones.

(Morin and Bengio, 2005) and negative sampling (Mikolov et al., 2013b). We adopt negative sampling not only because it is simpler and popular but also because it shows better performance as we see later.

\section{CASE STUDY}

Before diving into systematic evaluations, we provide two illustrative examples: Zachary's Karate club network and a word association network.

\section{Case Study: Zachary's Karate club network}

We use Zachary's Karate club network (Zachary, 1977), a well-known example for the community detection. Nodes represent members of the Karate club, and edges represent ties among the members (see Fig. 3a). Although it is often considered to have two large disjoint communities, smaller overlapping communities can also be seen, highlighted by nodes such as 1, 3, 28, and 32. In Fig. 3b, we present the persona graph of the network. persona $2 \mathrm{vec}$ successfully recognizes these bridge nodes and place their personas in reasonable places. Take node 1 for example. It splits into four persona nodes, which then end up in two different communities. The orange and green communities are clearly separated as a result. We also show the ten predictions with the highest score from the link prediction experiment in Fig. $3 \mathrm{~d}$ and checked that the model predicts missing edges well.

\section{Case Study: word association network}

Word association network captures how people associate words together (free association task). The dataset was originally assembled from nearly 750,000 responses from over 6,000 peoples. Participants were shown 5,019 words and asked to write down the first word that sprang in mind and all the word pairs were collected with their frequency as the weights. This dataset forms a weighted, directed graph of words that captures their multiple senses. Although it is, in principle, possible to run our method 


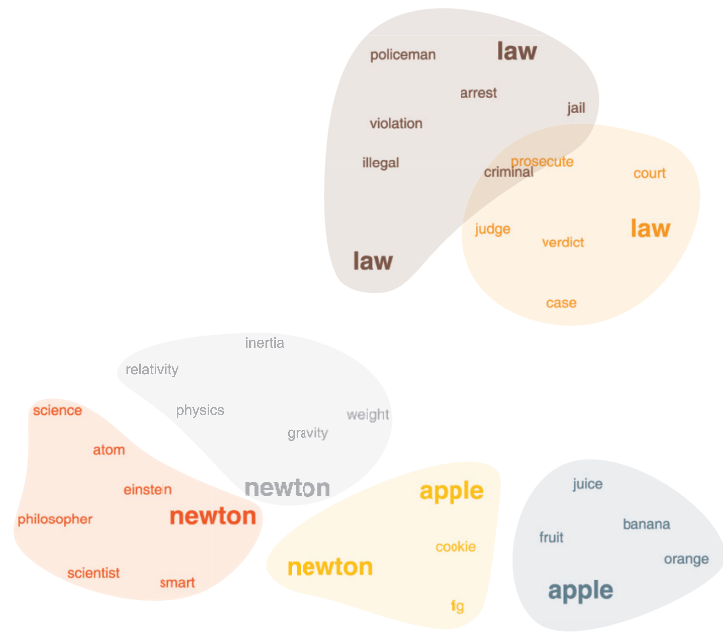

Figure 4. The word association network, clusters around the word "Newton". Coordinates of the words come from the 2-D projection of the embedding vectors with UMAP (McInnes et al., 2018). Word colors correspond to the clusters obtained by k-means clustering (MacQueen et al., 1967) on the embedding vectors.

on the original graph, for simplicity, we convert it into an undirected, unweighted graph by neglecting weight and direction (Ahn et al., 2010). In Fig. 4, we shows the persona2vec clusters around the word "Newton". We use the Louvain method (Blondel et al., 2008) to split the personas of each word. persona2vec successfully captures multiple contexts of the word "Newton". For instance, the red persona is associated with "scientists" and "philosopher", the grey one is linked to the physics, and the yellow one is associated with "apple" (note that there is a cookie called "(Fig) Newton" in the U.S.). Furthermore, persona2vec also captures different nuances of the word "law" that are related to the crime (brown cluster) and the legal concepts (orange cluster).

\section{NUMERICAL EXPERIMENT}

\section{Link Prediction Task}

To systematically evaluate the performance and scalability of the persona $2 \mathrm{vec}$ framework, we perform a link prediction task using real-world graphs (Grover and Leskovec, 2016; Abu-El-Haija et al., 2017). Link prediction aims to predict missing edges in a graph with partial information, which is useful for many tasks such as suggesting new friends on social networks or recommending products. It has been employed as a primary task to evaluate the performance of unsupervised graph embedding methods (Abu-El-Haija et al., 2017; Zhang et al., 2018).

We follow the task setup from the literature (Grover and Leskovec, 2016; Abu-El-Haija et al., 2017). First, the edge set of an input graph is divided equally and randomly into $E_{\text {train }}$ and $E_{\text {test }}$. We then refine $E_{\text {test }}$ using a rejection sampling based on the criterion that, even when we remove all edges in $E_{\text {test }}$, the graph should be connected as a single component. $E_{\text {train }}$ is used to train the models, and $E_{\text {test }}$ is used as positive examples for the prediction task. Second, a negative edge set $E_{(-)}$of non-existent random edges with the same size of $E_{\text {test }}$ are generated as negative examples for testing. The performance of a model is measured by its ability to correctly distinguish $E_{\text {test }}$ and $E_{(-)}$after being trained on $E_{\text {train }}$. We then report ROC-AUC.

\section{Datasets}

To facilitate the comparison with the state-of-the-art baseline, we use five graph datasets that are publicly available and previously used (Epasto and Perozzi, 2019). We summarize them as follows.

PPI is a protein-protein interaction graph of Homo sapiens (Stark et al., 2006). Nodes represent proteins and edges represent physical interactions between the proteins. ca-HepTh is a scientific collaboration graph. It represents the co-authorship among researchers from the Theoretical High Energy Physics field, derived from papers on arXiv. ca-AstropPh is also scientific collaboration graph, but from Astrophysics. 
wiki-vote is a voting network, each node is a Wikipedia user and a directed edge from node $i$ to node $j$ represents that user $i$ voted for user $j$ to become an administrator. soc-epinions is a voting graph from a general consumer review site Epinions. com, each node is a member, and a directed edge from node $i$ to node $j$ means that member $i$ trusted member $j$.

We use the largest component of the undirected graphs and the largest weakly connected component of the directed ones. The statistics of all the graphs are reported in Table 1.

\section{Methods}

The state-of-the-art method in this link prediction task is SPLITTER (Epasto and Perozzi, 2019), which also models multiple roles. As reported in the paper, it outperforms various exiting algorithms ranging across non-embedding methods like Jaccard Coefficient, Common Neighbors, and Adamic-Adar as well as embedding methods like Laplacian EigenMaps (Belkin and Niyogi, 2002), node2 vec (Grover and Leskovec, 2016), DNGR (Cao et al., 2016), Asymmetric (Abu-El-Haija et al., 2017) and M-NMF (Wang et al., 2017).

Given the state-of-the-art performance of SPLITTER, for simplicity, we compare our framework with SPLITTER using the identical task setup and datasets. In addition, because our method can be considered as an augmentation of a single-role embedding method, and because we use Node2 $\mathrm{vec}$ as the base embedding method, we also employ Node $2 \mathrm{vec}$. We run the link prediction task using the original authors' implementation of Node2 vec and SPLITTER. The parameters are also kept consistent with the original paper.

persona2vec and SPLITTER have multiple representations on each node, which leads to nonunique similarity estimations between two nodes. Hence, we define the similarity score of a pair of nodes on persona $2 \mathrm{vec}$ as the maximum dot-product of embedding vectors between any pair of their personas. We found that, among experiment with three aggregation functions min, max, mean, the highest performance is achieved with max, same with SPLITTER (Epasto and Perozzi, 2019). For SPLITTER, we use maximum cosine similarity, following the author's note in their implementation.

Node2vec (baseline method) For Node2vec, we set random walk length $t=40$, the number of walks per node $\gamma=10$, random walk parameters $p=q=1$, the window size $w=5$, and the initial learning rate $\alpha=0.025$. In the original paper, they learn an additional logistic regression classifier over the Hadamard product of the embedding of two nodes for the link prediction. In general, the logistic regression classifier improves the performance. Here, we report results on Node2 vec with both dot products and the logistic regression classifier.

SPLITTER (baseline method) For SPLITTER, we use the same parameters in their paper (Epasto and Perozzi, 2019) and Node2 vec baseline. We use node2vec with random walk parameters $p=q=1$.

persona2vec (our proposed method) We set the hyper-parameters of the original graph embedding with $t_{b}=40, \gamma_{b}=10, w_{b}=5$. For the persona embedding, we set $t_{p}=80, \gamma_{p}=5, w_{p}=2$ to better capture the micro-structure of the persona graph. The size of the total trajectories is determined by the random walk length $t_{*}$ times the number of walks per node $\gamma_{*}$, so we keep $t_{*} \gamma_{*}$ constant to roughly preserve the amount of information used in the embedding. For both embedding stages, we use the $\alpha=0.025$, and node2 vec with the random walk parameters $(p=q=1)$ as the graph embedding function.

\section{Experiment Results}

Fig. 5 shows the link prediction performance of persona2vec in comparison with the baselines. Overall, persona2vec yields superior performance across graphs and across a range of hyperparameter choice. We show that augmenting Node $2 \mathrm{vec}$ by considering personas significantly improves the link prediction performance, evinced by the significant performance gain (see Table 2).

As expected, larger dimensions lead to better performance, although persona2vec achieves reasonable results even with tiny embedding dimensions like 8 or 16 . We also show how the performance of persona2vec varies with $\lambda$. For undirected graphs, larger $\lambda$ is beneficial but the trend saturates quickly. For directed graphs, however, optimal performance is achieved with smaller values of $\lambda$. In practice, we suggest starting with $\lambda=0.5$ as a default parameter because the overall variation brought by $\lambda$ is not substantial and even when the performance increases with $\lambda$, near-optimal performance can be achieved at $\lambda=0.5$. 

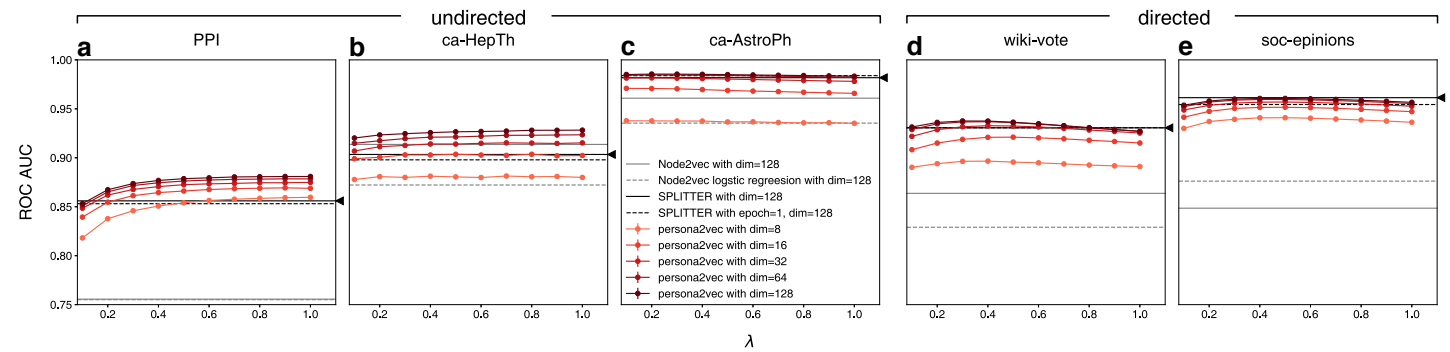

Figure 5. Performance of persona2vec in the link prediction task Number of epochs $n$ is set to 1 in all experiments for persona2vec. Darker colors represent higher embedding dimensions. The confidence intervals are all within the range of the markers. Given the same number of dimensions, persona2vec is always on par or better than SPLITTER

Table 2. Performance of persona2vec with $\lambda=0.5$. All methods use $d=128$. Node2vec* refers Node2vec with the logistic regression classifier, SPLITTER* refers SP LITTER with one epoch, and persona $2 \mathrm{vec}^{*}$ refers persona2vec with $\lambda=0.5$, our suggested default. Performance gain is performance difference between Node2vec and persona2 $\mathrm{vec}^{*}$. We omit the standard error which is smaller than $10^{-3}$.

\begin{tabular}{lrrrrr}
\hline Method & PPI & ca-HepTh & ca-AstroPh & wiki-vote & soc-epinions \\
\hline Node2vec & 0.585 & 0.825 & 0.901 & 0.694 & $0.547 \pm 0.007$ \\
Node2vec* & $0.662 \pm 0.001$ & 0.848 & 0.914 & $0.705 \pm 0.001$ & $0.767 \pm 0.002$ \\
SPLITTER & 0.856 & 0.903 & 0.982 & 0.931 & $\mathbf{0 . 9 6 1} \pm \mathbf{0 . 0 0 1}$ \\
SPLITTER* & 0.853 & 0.898 & 0.984 & 0.931 & $0.954 \pm 0.001$ \\
persona2vec* & $\mathbf{0 . 8 7 9}$ & $\mathbf{0 . 9 2 7}$ & $\mathbf{0 . 9 8 5}$ & $\mathbf{0 . 9 3 6}$ & $\mathbf{0 . 9 6 1}$ \\
\hline Performance gain & 0.294 & 0.102 & 0.084 & 0.242 & $0.414 \pm 0.007$ \\
\hline
\end{tabular}

When compared with the SPLITTER baseline, persona2vec shows on par or better performances given the same embedding dimensions across a wide range of $\lambda$. We also report the performance summary for persona $2 \mathrm{vec}$ with $\lambda=0.5$ (our suggested default) compared with the best baselines in Table 2, which show that persona2vec outperforms the baseline consistently. Also, we report the "performance gains" from Node2vec, because we used Node2vec as the base embedding method and persona $2 \mathrm{vec}$ can be considered an augmentation or fine-tuning of the base Node2 vec vectors with local structural information. As shown, the persona-based fine-tuning significantly improved the performance.

Also, we show the performance of both methods across different approximations: hierarchical softmax and negative sampling in Fig. 6 . We also found that cosine similarity consistently yields a better result with hierarchical softmax and dot product yields a better result with negative sampling across all methods. So, we use cosine similarity for hierarchical softmax results and use dot product for negative sampling results. We checked that both methods work well across the optimization method. We found that persona2vec tends to perform better with negative sampling and SPLITTER with hierarchical softmax. Nevertheless, persona2vec yields the best performance consistently.

In addition to the performance of the link prediction task, we also report the execution time of persona2vec and SPLITTER to compare their scalabilities in practice (see Fig. 7). Note that the reported execution time is on the link-prediction task, with half of the edges removed from the original graph. SP LITTER runs the embedding procedures for 10 epochs by default in the original implementation, whereas persona $2 \mathrm{vec}$ only runs for one epoch. For a fair comparison, we also report the results of SPLITTER with one epoch of training. When being limited to only one epoch, SPLITTER's performance slightly suffers on three graphs while it goes up or stays stable for the other two.

Nevertheless, persona2vec is more efficient-39 to 58 times faster than SPLITTER with 10 epochs and five to eight times faster than SPLITTER with one epoch, while consistently outperforming both. The most likely reason behind the drastic difference is the overhead from the extra regularization 


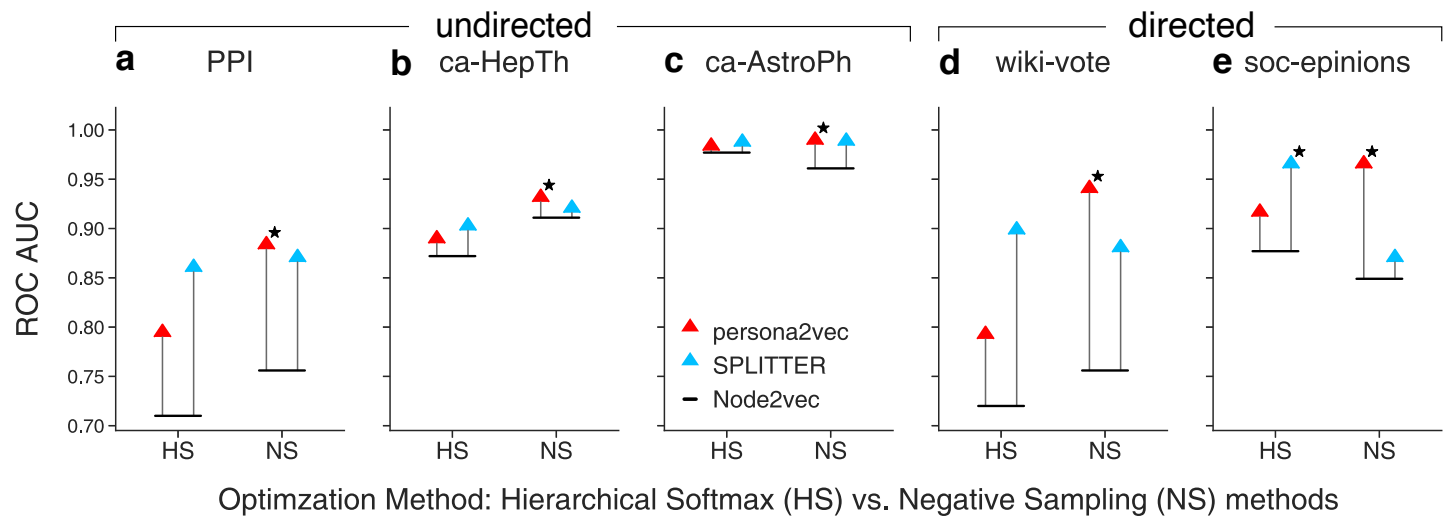

Figure 6. Comparison of link prediction performance between persona2vec and SPLITTER with different approximations. HS refers to the hierarchical softmax and NS refers to the negative sampling. The star marker indicates the best link prediction performance.

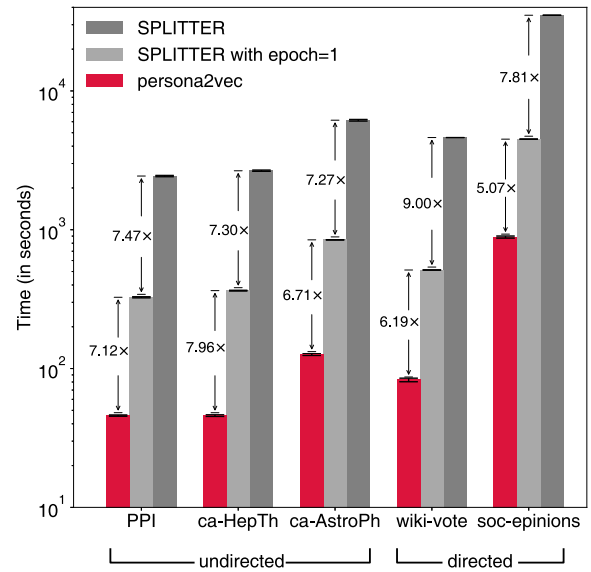

Figure 7. Comparison of elapsed time between persona2vec and SPLITTER. Speed gains by persona2vec are shown.

term in the cost function of SPLITTER, which persona2vec does not need. In sum, persona2vec outperforms the previous state-of-the-art method both in terms of scalability and link prediction performance.

\section{CONCLUSIONS}

We present persona2vec, a framework for learning multiple node representations considering the node's local structural contexts. persona2vec first performs ego-splitting, where nodes with multiple non-overlapping local communities in their ego-networks are replaced with corresponding persona nodes. The persona nodes inherit the edges from the original graph and remain connected by newly added persona edges, forming the persona graph. Initialized by the embedding of the original graph, the embedding algorithm applied to the persona graph yields the final representations. Instead of assigning only one vector to every node with multiple roles, persona 2 vec learns vectors for each of the personas. With extensive link prediction evaluations, we demonstrate that persona2 vec achieves the state-of-the-art performance while being able to scale better. Moreover, our method is easy to comprehend and implement without losing any flexibility for incorporating other embedding algorithms, presenting great potential for applications. The possible combination with various algorithms provides vast space for further exploration. For instance, in the multi-layer network, inter-layer coupling connection can be interpreted as natural persona edges, persona2 vec may be applied to tackle the multi-layer link prediction problem.

As we know, the graph (relational) structure is ubiquitous across many complex systems, including physical, social, economic, biological, neural, and information systems, and thus fundamental graph 
algorithms have far-reaching impacts across many areas of sciences. Graph embedding, in particular, removes the barrier of translating methods to the special graph data structure, opening up a powerful way to transfer existing algorithms to the graphs and relational data. Furthermore, given that it is natural to assume overlapping clusters and their heterogeneous functionality in most real networks, multi-role embedding methods may find numerous applications in physical, biological, and social sciences.

\section{ACKNOWLEDGMENTS}

For their comments, we thank Sadamori Kojaku, Alessandro Flammini, Filippo Menczer, Xiaoran Yan, Filipi Nascimento Silva, and Minwoo Ahn.

\section{Data Availability}

All datasets used in this paper are publicly available. For $P P I$, we use the prepossessed version from the node2 vec project web page (Grover and Leskovec, 2016), https://snap.stanford.edu/ node2 vec/Homo_sapiens.mat while other graphs are downloaded from the SNAP library homepage (Leskovec and Krevl, 2014), http: / / snap.stanford.edu/data/index.html (ca-AstroPh, caHepTh, wiki-Vote, soc-Epinions1).

\section{Code Availability}

Code used in this analysis can be found at https://github.com/jisungyoon/persona2vec

\section{REFERENCES}

Abu-El-Haija, S., Perozzi, B., and Al-Rfou, R. (2017). Learning edge representations via low-rank asymmetric projections. In Proceedings of the 2017 ACM on Conference on Information and Knowledge Management, CIKM '17, pages 1787-1796, New York, NY, USA. ACM.

Ahn, Y.-Y., Bagrow, J. P., and Lehmann, S. (2010). Link communities reveal multiscale complexity in networks. Nature, 466(7307):761-764.

Airoldi, E. M., Blei, D. M., Fienberg, S. E., and Xing, E. P. (2008). Mixed membership stochastic blockmodels. Journal of Machine Learning Research, 9(Sep):1981-2014.

Belkin, M. and Niyogi, P. (2002). Laplacian eigenmaps and spectral techniques for embedding and clustering. In Advances in Neural Information Processing Systems, pages 585-591.

Blondel, V. D., Guillaume, J.-L., Lambiotte, R., and Lefebvre, E. (2008). Fast unfolding of communities in large networks. Journal of Statistical Mechanics: Theory and Experiment, 2008(10):P10008.

Camacho-Collados, J., Pilehvar, M. T., and Navigli, R. (2016). Nasari: Integrating explicit knowledge and corpus statistics for a multilingual representation of concepts and entities. Artificial Intelligence, 240:36-64.

Cao, S., Lu, W., and Xu, Q. (2016). Deep neural networks for learning graph representations. In Thirtieth AAAI Conference on Artificial Intelligence.

Chen, H., Perozzi, B., Hu, Y., and Skiena, S. (2018). Harp: Hierarchical representation learning for networks. In Thirty-Second AAAI Conference on Artificial Intelligence, Hilton New Orleans Riverside, New Orleans, Louisiana, USA. AAAI press.

Chen, X., Liu, Z., and Sun, M. (2014). A unified model for word sense representation and disambiguation. In Proceedings of the 2014 Conference on Empirical Methods in Natural Language Processing (EMNLP), pages 1025-1035.

Cheng, J., Wang, Z., Wen, J.-R., Yan, J., and Chen, Z. (2015). Contextual text understanding in distributional semantic space. In Proceedings of the 24th ACM International on Conference on Information and Knowledge Management, pages 133-142. ACM.

Coscia, M., Rossetti, G., Giannotti, F., and Pedreschi, D. (2014). Uncovering hierarchical and overlapping communities with a local-first approach. ACM Trans. Knowl. Discov. Data, 9(1):6:1-6:27.

Epasto, A., Lattanzi, S., Mirrokni, V., Sebe, I. O., Taei, A., and Verma, S. (2015). Ego-net community mining applied to friend suggestion. Proceedings of the VLDB Endowment, 9(4):324-335.

Epasto, A., Lattanzi, S., and Paes Leme, R. (2017). Ego-splitting framework: From non-overlapping to overlapping clusters. In Proceedings of the 23rd ACM SIGKDD International Conference on Knowledge Discovery and Data Mining, KDD '17, pages 145-154, New York, NY, USA. ACM. 
Epasto, A. and Perozzi, B. (2019). Is a single embedding enough? learning node representations that capture multiple social contexts. In The World Wide Web Conference, pages 394-404, New York, NY, USA. ACM, ACM.

Evans, T. S. and Lambiotte, R. (2009). Line graphs, link partitions, and overlapping communities. Phys. Rev. E, 80:016105.

Fortunato, S. (2010). Community detection in graphs. Physics Reports, 486(3-5):75-174.

Gavin, A.-C., Aloy, P., Grandi, P., Krause, R., Boesche, M., Marzioch, M., Rau, C., Jensen, L. J., Bastuck, S., Dümpelfeld, Birgit, E. A., Heurtier, M.-A., Hoffman, V., Hoefert, C., Klein, K., Hudak, M., Michon, A.-M., Schelder, M., Schirle, M., Remor, M., Rudi, T., Hooper, S., Bauer, A., Bouwmeester, T., Casari, G., Drewes, G., Neubauer, G., Rick, J. M., Kuster, B., Bork, P., Russell, R. B., and Superti-Furga, G. (2006). Proteome survey reveals modularity of the yeast cell machinery. Nature, 440(7084):631.

Grover, A. and Leskovec, J. (2016). Node2vec: Scalable feature learning for networks. In Proceedings of the 22Nd ACM SIGKDD International Conference on Knowledge Discovery and Data Mining, KDD '16, pages 855-864, New York, NY, USA. ACM.

Iacobacci, I., Pilehvar, M. T., and Navigli, R. (2015). Sensembed: Learning sense embeddings for word and relational similarity. In Proceedings of the 53rd Annual Meeting of the Association for Computational Linguistics and the 7th International Joint Conference on Natural Language Processing (Volume 1: Long Papers), pages 95-105.

Jauhar, S. K., Dyer, C., and Hovy, E. (2015). Ontologically grounded multi-sense representation learning for semantic vector space models. In Proceedings of the 2015 Conference of the North American Chapter of the Association for Computational Linguistics: Human Language Technologies, pages 683-693.

Leskovec, J. and Krevl, A. (2014). SNAP Datasets: Stanford large network dataset collection. http: //snap.stanford.edu/data.

Leskovec, J., Lang, K. J., Dasgupta, A., and Mahoney, M. W. (2009). Community structure in large networks: Natural cluster sizes and the absence of large well-defined clusters. Internet Mathematics, 6(1):29-123.

Leskovec, J., Lang, K. J., and Mahoney, M. (2010). Empirical comparison of algorithms for network community detection. In Proceedings of the 19th International Conference on World Wide Web, WWW '10, pages 631-640, New York, NY, USA. ACM.

Li, J. and Jurafsky, D. (2015). Do multi-sense embeddings improve natural language understanding? preprint arXiv:1506.01070.

Liu, N., Tan, Q., Li, Y., Yang, H., Zhou, J., and Hu, X. (2019). Is a single vector enough? exploring node polysemy for network embedding. In Proceedings of the 25th ACM SIGKDD International Conference on Knowledge Discovery \& Data Mining, pages 932-940.

Liu, P., Qiu, X., and Huang, X. (2015a). Learning context-sensitive word embeddings with neural tensor skip-gram model. In Twenty-Fourth International Joint Conference on Artificial Intelligence.

Liu, Y., Liu, Z., Chua, T.-S., and Sun, M. (2015b). Topical word embeddings. In Twenty-Ninth AAAI Conference on Artificial Intelligence.

Maaten, L. v. d. and Hinton, G. (2008). Visualizing data using t-sne. Journal of Machine Learning Research, 9(Nov):2579-2605.

MacQueen, J. et al. (1967). Some methods for classification and analysis of multivariate observations. In Proceedings of the fifth Berkeley symposium on mathematical statistics and probability, volume 1, pages 281-297. Oakland, CA, USA.

McInnes, L., Healy, J., and Melville, J. (2018). Umap: Uniform manifold approximation and projection for dimension reduction. preprint arXiv:1802.03426.

Mikolov, T., Chen, K., Corrado, G., and Dean, J. (2013a). Efficient estimation of word representations in vector space. preprint arXiv:1301.3781.

Mikolov, T., Sutskever, I., Chen, K., Corrado, G. S., and Dean, J. (2013b). Distributed representations of words and phrases and their compositionality. In Advances in neural information processing systems, pages 3111-3119.

Morin, F. and Bengio, Y. (2005). Hierarchical probabilistic neural network language model. In Aistats, volume 5, pages 246-252. Citeseer.

Neelakantan, A., Shankar, J., Passos, A., and McCallum, A. (2015). Efficient non-parametric estimation of multiple embeddings per word in vector space. preprint arXiv:1504.06654. 
Palla, G., Derényi, I., Farkas, I., and Vicsek, T. (2005). Uncovering the overlapping community structure of complex networks in nature and society. Nature, 435(7043):814.

Pelevina, M., Arefyev, N., Biemann, C., and Panchenko, A. (2017). Making sense of word embeddings. preprint arXiv:1708.03390.

Pennington, J., Socher, R., and Manning, C. (2014). Glove: Global vectors for word representation. In Proceedings of the 2014 Conference on Empirical Methods in Natural Language Processing (EMNLP), pages 1532-1543.

Perozzi, B., Al-Rfou, R., and Skiena, S. (2014). Deepwalk: Online learning of social representations. In Proceedings of the 20th ACM SIGKDD International Conference on Knowledge Discovery and Data Mining, KDD '14, pages 701-710, New York, NY, USA. ACM.

Raghavan, U. N., Albert, R., and Kumara, S. (2007). Near linear time algorithm to detect community structures in large-scale networks. Physical review E, 76(3):036106.

Reisinger, J. and Mooney, R. J. (2010). Multi-prototype vector-space models of word meaning. In Human Language Technologies: The 2010 Annual Conference of the North American Chapter of the Association for Computational Linguistics, pages 109-117. Association for Computational Linguistics.

Rossi, R. A. and Ahmed, N. K. (2015). The network data repository with interactive graph analytics and visualization. In $A A A I$.

Rosvall, M. and Bergstrom, C. T. (2008). Maps of random walks on complex networks reveal community structure. Proceedings of the National Academy of Sciences, 105(4):1118-1123.

Rosvall, M., Esquivel, A. V., Lancichinetti, A., West, J. D., and Lambiotte, R. (2014). Memory in network flows and its effects on spreading dynamics and community detection. Nature Communications, 5:4630.

Rothe, S. and Schütze, H. (2015). Autoextend: Extending word embeddings to embeddings for synsets and lexemes. preprint arXiv:1507.01127.

Sen, P., Namata, G., Bilgic, M., Getoor, L., Galligher, B., and Eliassi-Rad, T. (2008). Collective classification in network data. AI magazine, 29(3):93-93.

Stark, C., Breitkreutz, B.-J., Reguly, T., Boucher, L., Breitkreutz, A., and Tyers, M. (2006). Biogrid: a general repository for interaction datasets. Nucleic Acids Research.

Tang, J., Qu, M., Wang, M., Zhang, M., Yan, J., and Mei, Q. (2015). Line: Large-scale information network embedding. In Proceedings of the 24th International Conference on World Wide Web, WWW '15, pages 1067-1077, Republic and Canton of Geneva, Switzerland. International World Wide Web Conferences Steering Committee.

Wang, X., Cui, P., Wang, J., Pei, J., Zhu, W., and Yang, S. (2017). Community preserving network embedding. In Thirty-First AAAI Conference on Artificial Intelligence.

Wu, Z. and Giles, C. L. (2015). Sense-aaware semantic analysis: A multi-prototype word representation model using wikipedia. In Twenty-Ninth AAAI Conference on Artificial Intelligence.

Xie, G.-S., Liu, L., Zhu, F., Zhao, F., Zhang, Z., Yao, Y., Qin, J., and Shao, L. (2020). Region graph embedding network for zero-shot learning. In European Conference on Computer Vision, pages 562-580. Springer.

Yang, L., Cao, X., He, D., Wang, C., Wang, X., and Zhang, W. (2016). Modularity based community detection with deep learning. In IJCAI, volume 16, pages 2252-2258, New York, New York, USA. AAAI Press.

Zachary, W. W. (1977). An information flow model for conflict and fission in small groups. Journal of Anthropological Research, 33(4):452-473.

Zhang, H. and Zhong, G. (2016). Improving short text classification by learning vector representations of both words and hidden topics. Knowledge-Based Systems, 102:76-86.

Zhang, Z., Cui, P., Wang, X., Pei, J., Yao, X., and Zhu, W. (2018). Arbitrary-order proximity preserved network embedding. In Proceedings of the 24th ACM SIGKDD International Conference on Knowledge Discovery \& Data Mining, pages 2778-2786. ACM. 